Book Comment

\title{
The Pharmacist Guide
}

\section{to Implementing Pharmaceutical Care}

\author{
F. Alves da Costa, J.W.F. van Mil, A. Alvarez-Risco, Editors \\ Springer, New York, 2018. \\ Hard cover: ISBN 978-3-319-92575-2 \\ eBook: ISBN 978-3-319-92576-9
}

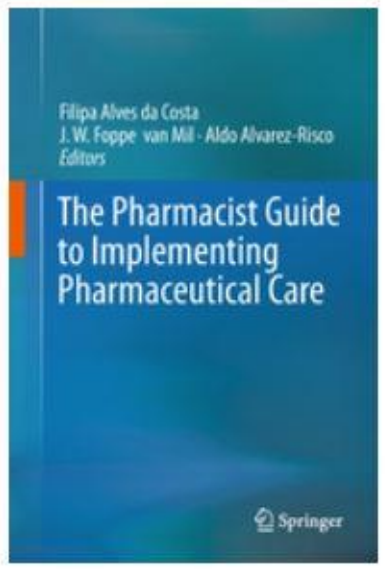

The Pharmacist Guide to Implementing Pharmaceutical Care is published by Springer and focuses on the implementation of pharmaceutical care. This book provides an in-depth analysis of particularities in care recipients and care environment impacting on service provision, complemented with practical examples. This book is aimed at pharmacists and pharmacy students, and edited by three experts in pharmaceutical care.

The book is structured in eight parts, covering 40 chapters:

Part I: What is Pharmaceutical Care, and Part II: Pharmaceutical Care Processes, discuss the pillars of pharmaceutical care, including the philosophy of practice, the central aim of the service focused around the identification and solving of drug related problems, the documentation needed, the indicators to monitor the process and the outcomes for the service beneficiaries and aspects of inter-professional collaboration. Specific services within pharmaceutical care are highlighted, such as contributing to medication adherence, providing medication review, and medication reconciliation.

Part III: Pharmaceutical Care around the World provides an overview of practice and research around five continents, aiming to enable the identification of crucial aspects of implementation that might impact of transferability of concepts.

Fernando FERNANDEZ-LLIMOS. PhD, PharmD, MBA Editor-in-chief, Pharmacy Practice. Institute for Medicines Research (iMed.ULisboa), Department of Social Pharmacy, Faculty of Pharmacy, Universidade de Lisboa. Lisbon (Portugal)
Part IV: Implementing Pharmaceutical Care in Different Settings starts by focusing on general implementation strategies, followed by specific aspects related to the setting, highlighting aspects relevant to community pharmacy, hospitals and clinics, and finally nursing homes.

Part V: Delivering Pharmaceutical Care in Practice focuses on structural and complementary aspects of pharmaceutical care, and is divided into health promotion and disease prevention, dispensing medicines, pharmaceutical care around OTC medication and around medical devices.

Part VI: Pharmaceutical Care for Specific Patient Groups details the general aspects covered in part I considering the particularities of the medical condition debated, covering non-communicable diseases, such as asthma, diabetes, cardiovascular diseases and oncology, to name a few, but also communicable diseases, such as viral diseases, including Hepatitis and HIV.

Part VII: Remuneration of Pharmaceutical Care provides the readers with basic economy concepts applied to health research, expanding then to remuneration models in general and in pharmacy practice in particular.

Part VIII: Teaching Pharmaceutical Care is a part particularly intended for educators, both working in academia and in pharmacy practice focusing on professional continuous development. This part provides a detailed overview of teaching methods, also providing practical examples of curricular restructuring aimed at alignment with practice.

The book was developed with the contribution of 67 authors from all continents, selected as experts in the different fields of practice. Additionally, 17 reputed researchers contributed by reviewing the chapters in an external peer-review process.

The book is really comprehensive, and very useful for everyone willing to start implementing pharmaceutical care, or improving the success of implementation. It is an exhaustive book which can efficiently guide the implementation of enhanced person-centred care. Like every book, it reflects the current situation, and hopefully the publisher will make sure it is updated regularly. 